\title{
DEFINING SARCOPENIA USING MUSCLE QUALITY INDEX
}

\author{
C.-D. Lee, E. Dierickx
}

\begin{abstract}
Objectives: Although low muscle quality is a strong predictor of sarcopenia, defining sarcopenia using muscle quality remains unknown. This study investigated the cut-points to define sarcopenia using muscle quality index (MQI) in the young reference population. Methods: Fifty healthy young (20 to 29 years) and forty elderly adults (60 to 79 years) were recruited in this study. Dual-energy X-ray absorptiometry was used to assess appendicular skeletal muscle mass. Hand grip and leg dynamometers were used to measure muscle strengths in the arm and leg. Muscle quality in the arm (MQI $\left.{ }_{\text {Arm }} \mathrm{kg} / \mathrm{kg}\right)$ and leg $\left(\mathrm{MQI}_{\mathrm{Leg}^{\prime}} \mathrm{Nm} / \mathrm{kg}\right.$ ) were computed as muscle strength per lean mass in the arm and leg, respectively. Total muscle quality $\left(\mathrm{MQI}_{\mathrm{Total}}\right)$ was computed as the combination of $\mathrm{MQI}_{\mathrm{Arm}}$ and $\mathrm{MQI}_{\mathrm{Leg}^{\prime}}$ while standardized muscle quality $\left(\mathrm{MQI}_{\mathrm{Std}}\right)$ was computed as the combination of $\mathrm{z}$-scores in $\mathrm{MQI}_{\mathrm{Arm}}$ and $\mathrm{MQI} \mathrm{L}_{\mathrm{Leg}}$. Sarcopenia was defined as $\leq 2 \mathrm{SD}$ below from the mean values in the young reference group. Results: The cut-points for defining sarcopenia using $\mathrm{MQI}_{\mathrm{Arm}^{\prime}} \mathrm{MQI}_{\mathrm{Leg}^{\prime}} \mathrm{MQI}_{\mathrm{Tota}}$ and $^{\mathrm{M}} \mathrm{MQI} \mathrm{Std}$ in $\mathrm{men}$ were $\leq 8.37$, $\leq 12.07,22.06$, and $<-3.35$, and in women were $\leq 10.09, \leq 13.97,28.22$, and $<-2.25$, respectively. In the elderly adults, the frequencies of sarcopenia using $\mathrm{MQI}_{\mathrm{Arm}^{\prime}} \mathrm{MQI}_{\mathrm{Leg}^{\prime}} \mathrm{MQI}_{\mathrm{Tota}{ }^{\prime}}$ and $\mathrm{MQI}_{\mathrm{Std}}$ were $15 \%, 27.5 \%, 32.5 \%$, and 35\%, respectively. Conclusion: This study establishes new values for defining sarcopenia using MQIs. The proposed new MQI cut-points may be a role in detecting sarcopenia across individual and population level.
\end{abstract}

Key words: sarcopenia, muscle quality index, muscle mass, muscle strength.

\section{Introduction}

Age-related skeletal muscle loss (sarcopenia) is a significant risk factor for falls (1), disability (2), and mortality (3) in the elderly men and women. With increasing life expectancy, sarcopenia is a major public health concern in the geriatric societies (4). Approximately 7 to $27.8 \%$ of the United States (US) men and 10 to $19.3 \%$ of US women, aged $\geq 60$ years, suffer from sarcopenia (5-6), with increasing prevalence of sarcopenia in persons over 80 years of age $(50 \%)(7)$ and in some cancer patients (68.9\%) (8). Estimated medical costs associated with sarcopenia in the US is about 18 billion a year (5). Since effective clinical treatments for sarcopenia in the elderly populations are limited, preserving muscle mass and muscle strength at younger and middle ages is imperative to avoid the burden of this disease.

Skeletal muscle mass decreases about $40 \%$ between the ages of 20 and 80 years (9) with an annual decreasing rate of 1 to $2 \%$ after 50 years of age (10). Notably, persons with skeletal muscle loss and excess fat are at greater risk of physical disability and mortality (11). Although the primary causes of sarcopenia still remain unknown, early

School of Nutrition and Health Promotion, Arizona State University, Phoenix, AZ;

Corresponding Author: Dr. Chong Lee, Arizona State University, School of Nutrition and Health Promotion, 500 North 3rd street, Phoenix, AZ 85004, Phone: 602-827-2282, Fax: 602-496-1873, Email: chong.lee@asu.edu detection and subsequent treatment of sarcopenia is a key to prevent sarcopenia-related disability and mortality.

To address sarcopenia prevention strategies, establishing an accurate definition of sarcopenia should be in the first place. Baumgartner et al. first proposed cutpoints to define sarcopenia using low muscle mass (12). Several investigators have shown that low muscle mass is associated with disability $(2,12)$ and mortality $(13)$. Other investigators have also shown that low muscle strength, not low muscle mass, is associated with disability (14) and mortality (15). Currently, sarcopenia has been defined as the combination of low muscle mass and weakness or slowness (16-20). However, the definition of sarcopenia still remains in dispute worldwide. The European Working Group on Sarcopenia in Older People (EWGSOP) (16) and the Asian Working Group for Sarcopenia (AWGS) consensus panels have defined sarcopenia using the combination of low muscle mass and low muscle function (17). The International Working Group on Sarcopenia (IWGS) (18) and the Society of Sarcopenia, Cachexia and Wasting Disorders (SCWD) (19) have defined sarcopenia using the combination of low muscle mass and low physical performance. The Foundation for the National Institutes of Health (FNIH) (20) defined sarcopenia using the combination of low muscle mass and low muscle strength. These inconsistent guidelines may lead to confusion to the public and clinical settings as a screening tool to determine sarcopenia patients. 
From the methodological perspectives, the definition of sarcopenia should represent muscle quality, muscle strength per unit of muscle mass, rather than muscle mass or muscle strength. Some investigators have shown that intermuscular fat increases by $35.5-74.6 \%$ in men and $16.8-50 \%$ in women with aging (21), and an increase in fat mass is positively associated with muscle mass and muscle strength (22). Thus, the greater muscle strength or muscle mass associated with increment in fat may lack muscle quality. Although few studies have shown that muscle quality is a better indicator of functional capacity as compared with muscle mass or muscle strength (23), there is no standardized method to define sarcopenia using muscle quality. To fill this gap, we investigated the cut-points to define sarcopenia using the muscle quality indexes (MQIs) in the young reference adults.

\section{Methods}

\section{Study Participants}

Fifty young male and female adults (ages 20-29 years; $\mathrm{m}=30, \mathrm{f}=20$ ) and forty elderly male and female adults (ages 60 to 79 years; $\mathrm{m}=16 ; \mathrm{f}=24$ ) were recruited for the present study. The study was advertised by fliers, online posts, and University announcements within the Downtown Phoenix area. For the healthy young reference group, inclusion criteria were aged 20 to 29 years, body mass index (BMI) $<30 \mathrm{~kg} / \mathrm{m}^{2}$ (weight in kilograms divided by height in meters squared), ability to perform physical activity assessed by online physical activity readiness questionnaire (PAR-Q), no pregnant, no personal history of chronic diseases, and not taking any hypoglycemic and hypertensive medications. For the elderly persons, inclusion criteria were aged 60 or more, ability to perform physical activity assessed by online PAR-Q, with no personal history of heart disease, stroke, or cancer. Written informed consent was obtained from all subjects prior to study participation. The study was approved by the Institutional Review Board at Arizona State University. All participants were given a detailed description of the protocol prior to their participation.

\section{Measurement Procedure}

Body height and weight were measured using a standardized physician's scale. Dual-energy X-ray absorptiometry (DXA) was used to assess body composition, and arm and leg skeletal muscle mass by a licensed technician (Lunar iDXA, GE Healthcare, Madison, WI). Appendicular skeletal muscle mass (SMS) was computed by combining lean tissues in both arms and legs, and relative muscle mass was computed as SMS divided by height in meters squared (AMS) or SMS divided by body mass index ( $\mathrm{AMS}_{\mathrm{BMI}}$ ).

The grip strength was measured using Takei Physical Fitness Test dynamometer $(\mathrm{kg})$. The dominant hand was used with the subject standing and their arm at a position parallel to the floor. Grip strength was measured twice, and the average of two test scores was used for analysis. The leg strength was measured by isometric knee extension test ( 1 set of 3 repetitions) at an angle of 60 degrees using the CSMI Humac Norm Dynamometer test $(\mathrm{Nm})$. An average of the highest two performance scores was used for analysis.

Muscle quality in the arm $\left(\mathrm{MQI}_{\mathrm{Arm}^{\prime}} \mathrm{kg} / \mathrm{kg}\right)$ was calculated as the grip strength $\left(\mathrm{STR}_{\mathrm{Arm}^{\prime}} \mathrm{kg}\right)$, right arm, divided by the lean mass in the right arm $\left(\mathrm{LM}_{\mathrm{Arm}}\right.$, $\mathrm{kg})$. Muscle quality in the leg $\left(\mathrm{MQI}_{\mathrm{Leg}^{\prime}} \mathrm{Nm} / \mathrm{kg}\right)$ was calculated as the isometric leg strength $\left(\mathrm{STR}_{\mathrm{Leg}^{\prime}} \mathrm{Nm}\right)$, right leg, divided by lean mass in the right leg $\left(\mathrm{LM}_{\mathrm{Leg}}\right.$, $\mathrm{kg})$. Total muscle quality (MQI $\mathrm{Iotal}_{1}$ ) was computed as the combination of $\mathrm{MQI}_{\mathrm{Arm}}$ and $\mathrm{MQI} \mathrm{Leg}_{\text {. Standardized MQI }}$ $\left(\mathrm{MQI}_{\mathrm{Std}}\right)$ was computed as the combination of z-scores in both MQI ${ }_{\text {Arm }}$ and MQI ${ }_{\text {Leg: }}$. In the elderly persons, $\mathrm{MQI}_{\mathrm{Std}}$ was computed by the combination of z-scores in both $M Q I_{A r m}$ and MQI ${ }_{L e g}$ using the sex-specific means and SDs from the healthy young reference group.

\section{Statistical Analysis}

General linear models were used to investigate mean differences for anthropometric, clinical measures, relative muscle mass and muscle quality indexes between men and women after adjustment for age and race. The normality assumptions for all outcome measures were justified by Shapiro-Wilk test or Kolmogorov-Smirnov test. Sex-specific cut-points for ASM, $\mathrm{ASM}_{\mathrm{BMI}}, \mathrm{MQI} \mathrm{Arm}_{\mathrm{Ar}^{\prime}}$ $\mathrm{MQI}_{\mathrm{Leg}^{\prime}} \mathrm{MQI}_{\text {Total }}$ and MQI $\mathrm{Std}_{\text {td }}$ were computed as $>1 \mathrm{SD}, 1$ $\mathrm{SD} \geq$ to $>2 \mathrm{SD}$, and $\leq 2 \mathrm{SD}$ below from the mean values in the young reference group. Sarcopenia was defined as $\leq 2$ $\mathrm{SD}$ below from the mean values in the young reference group. We also examined the sex- and race-adjusted partial Pearson correlations among of muscle mass, muscle strength, and muscle quality in both young and elderly adults, respectively. All statistical procedures were performed by Statistical Analysis Systems (SAS 9.4) software (SAS Institute, Cary, NC).

\section{Results}

In the young reference group, as shown in Table 1, men had greater BMI, SBP, grip strength, STR $\mathrm{Leg} \mathrm{LM}_{\mathrm{Arm}^{\prime}}$ and $\mathrm{LM}_{\mathrm{Leg}}$ than did women after adjustment for age and race (all $\mathrm{p}<0.001$ ). There were no statistical gender differences in $\mathrm{DBP}, \mathrm{MQI}_{\mathrm{Leg}^{\prime}} \mathrm{MQI}_{\text {Total }}$ and $\mathrm{MQI}_{\mathrm{Std}}$ (all $\mathrm{p}>0.10$ ), while women had greater $M Q I_{A r m}$ than did men $(p<0.001)$. In the elderly persons, men had greater grip strength, $\mathrm{LM}_{\mathrm{Arm}}$ $\mathrm{STR}_{\mathrm{Leg}^{\prime}} \mathrm{LM}_{\mathrm{Leg}^{\prime}} \mathrm{MQI}_{\mathrm{Std}}$ than did women (all $\mathrm{p}<0.02$ ). There were no statistical gender differences in BMI, SBP, DBP, $M Q I_{\mathrm{Arm}^{\prime}} \mathrm{MQI}_{\mathrm{Leg}^{\prime}}$ and MQI $\mathrm{Total}$ (all $\mathrm{p}>0.35$ ).

The sex-specific relative muscle mass (ASM and $\mathrm{ASM}_{\mathrm{BMI}}$ ) and MQI (MQI $\mathrm{Arm}, \mathrm{MQI}_{\mathrm{Leg}^{\prime}}, \mathrm{MQI}_{\mathrm{Total}}$, and MQI $\mathrm{Std}$ ) cut-points are shown in Table 2. The ASM, ASM ${ }_{\mathrm{BMI}^{\prime}}$ and 
Table 1

Characteristics of the study participants in young and elderly adults

\begin{tabular}{lcccccc}
\hline & \multicolumn{3}{c}{ Young Adults } & \multicolumn{3}{c}{ Elderly Adults } \\
Variables & Men & Women & P-value & Men & Women & P-value \\
\hline Age $(\mathrm{y})^{*}$ & 23.7 & 23.1 & & 69.4 & 68.1 & \\
Race $($ white, $\%)$ & 57 & 80 & & 81 & 83 & \\
Adjusted valuest & & & & & & $<0.001$ \\
Height $(\mathrm{cm})$ & 179.8 & 166.7 & $<0.001$ & 174.1 & 161.7 & 0.09 \\
Weight $(\mathrm{kg})$ & 82.2 & 62.6 & $<0.001$ & 85.8 & 74.2 & 0.94 \\
BMI $\left(\mathrm{kg} / \mathrm{m}^{2}\right)$ & 25.4 & 22.5 & $<0.001$ & 28.3 & 28.2 & 0.58 \\
SBP $(\mathrm{mmHg})$ & 124.4 & 112.8 & $<0.001$ & 131.4 & 128.5 & 0.42 \\
DBP $(\mathrm{mmHg})$ & 71.8 & 69.3 & 0.24 & 76.2 & 74.1 & $<0.001$ \\
Grip Strength $(\mathrm{kg})$ & 54.61 & 34.44 & $<0.001$ & 41.8 & 25.3 & $<0.001$ \\
LM $_{\text {Arm }}(\mathrm{kg})$ & 4.64 & 2.50 & $<0.001$ & 3.57 & 2.17 & $<0.001$ \\
STR $_{\text {Leg }}(\mathrm{N} \mathrm{m})$ & 231.1 & 152.0 & $<0.001$ & 150.6 & 99.5 & $<0.001$ \\
LM $_{\text {Leg }}(\mathrm{kg})$ & 10.76 & 7.48 & $<0.001$ & 9.09 & 6.75 & 0.91 \\
MQI $_{\text {Arm }}$ & 11.8 & 13.9 & $<0.001$ & 11.9 & 12.0 & 0.35 \\
MQI $_{\text {Leg }}$ & 21.9 & 20.6 & 0.32 & 16.9 & 15.5 & 0.49 \\
MQI $_{\text {Total }}$ & 33.7 & 34.5 & 0.57 & 28.8 & 27.5 & 0.02 \\
MQI $_{\text {Std }}$ & -0.29 & 0.44 & 0.10 & -0.94 & -2.55 & \\
\hline
\end{tabular}

*Values are means. + Adjusted for age and race. $\mathrm{LM}_{\mathrm{Arm}}$ a lean mass in the arm; $\mathrm{LM}_{\mathrm{Leg}}$ a lean mass in the leg; $\mathrm{STR}_{\mathrm{Leg}}$ leg strength; $\mathrm{MQI}_{\mathrm{Arm}}$ muscle quality in the arm;

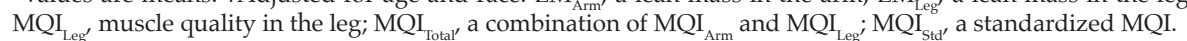

MQI cut-points were classified as normal, low, and poor categories, corresponding to $>1 \mathrm{SD}, 1 \mathrm{SD} \geq$ to $>2 \mathrm{SD}$ and $\leq 2 \mathrm{SD}$ below from the sex-specific mean values in the young reference group. "Poor" categories were classified as sarcopenia. The cut-points for sarcopenia using ASM and $\mathrm{ASM}_{\mathrm{BMI}}$ in men were $\leq 7.75 \mathrm{~kg} / \mathrm{m}^{2}$ and $\leq 0.96$, and in women were $\leq 5.69 \mathrm{~kg} / \mathrm{m}^{2}$ and $\leq 0.71$, respectively. The cut-points for sarcopenia using $\mathrm{MQI} \mathrm{Arm}_{\mathrm{Arm}^{\prime}} \mathrm{MQI}_{\mathrm{Leg}^{\prime}} \mathrm{MQI}_{\mathrm{Total}}$ and $\mathrm{MQI}_{\mathrm{Std}}$ in men were $\leq 8.37, \leq 12.07,22.06$, and $<-3.35$, and in women were $\leq 10.09, \leq 13.97,28.22$, and $<-2.25$, respectively.

In the elderly adults, the frequencies of sarcopenia using $\mathrm{MQI}_{\mathrm{Arm}^{\prime}}, \mathrm{MQI} \mathrm{Leg}_{\mathrm{Leg}^{\prime}} \mathrm{MQI}_{\mathrm{Total}}$ and $\mathrm{MQI}_{\mathrm{Std}}$ were $15 \%$ $(\mathrm{n}=6), 27.5 \%(\mathrm{n}=11), 32.5 \%(\mathrm{n}=13)$, and $35 \%(\mathrm{n}=14)$, respectively. The sex- and race-adjusted partial Pearson correlations among muscle mass, muscle strength, and muscle quality are shown in Table 3. There was no association between muscle mass and muscle strength in both young $(\mathrm{r}=0.19, \mathrm{p}=0.20)$ and elderly adults $(\mathrm{r}=$ $0.05, \mathrm{p}=0.75)$, but muscle mass was inversely associated with muscle quality in young $(\mathrm{r}=-0.48, \mathrm{p}<0.001)$ and elderly adults $(\mathrm{r}=-0.73, \mathrm{p}<0.001)$. There was a moderate association between muscle strength and muscle quality in young $(r=0.62, p<0.001)$ and elderly persons $(r=0.46$, $\mathrm{p}<0.001)$.
Table 2

Cut-points to define sarcopenia using relative muscle mass and muscle quality indexes in young men and women

\begin{tabular}{lccc}
\hline & \multicolumn{3}{c}{ Sarcopenia Cut-points* } \\
Characteristic & Normal & Low & Poor \\
\hline Men $(\mathrm{N}=30)$ & 8.61 & $8.61-7.75$ & 7.75 \\
$\mathrm{ASM}$ & 1.08 & $1.08-0.96$ & 0.96 \\
$\mathrm{ASM}_{\text {BMI }}$ & 10.13 & $10.13-8.37$ & 8.37 \\
MQI $_{\text {Arm }}$ & 16.71 & $16.71-12.07$ & 12.07 \\
MQI $_{\text {Leg }}$ & 27.65 & $27.65-22.06$ & 22.06 \\
MQI $_{\text {Total }}$ & -1.68 & -1.68 to -3.35 & -3.35 \\
MQI $_{\text {Std }}$ & & & \\
Women $(\mathrm{N}=20)$ & 6.32 & $6.32-5.69$ & 5.69 \\
ASM & 0.79 & 0.79 to 0.71 & 0.71 \\
ASM $_{\text {BMI }}$ & 11.95 & $11.95-10.09$ & 10.09 \\
MQI $_{\text {Arm }}$ & 17.68 & $17.68-13.97$ & 13.97 \\
MQI $_{\text {Leg }}$ & 31.70 & $31.70-28.22$ & 28.22 \\
MQI $_{\text {Total }}$ & -1.12 & -1.12 to -2.25 & -2.25 \\
MQI $_{\text {Std }}$ & &
\end{tabular}

${ }^{*}$ Normal, low, and poor indicates $>1 \mathrm{SD}, 1 \mathrm{SD} \geq$ to $>2 \mathrm{SD}$, and $\leq 2 \mathrm{SD}$ below from the sex-specific mean values in the reference group. ASM, appendicular skeletal muscle mass; $\mathrm{ASM}_{\mathrm{BMI}^{\prime}}$ ASM divided by height in meters squared; $\mathrm{MQI}_{\mathrm{Arm}}$ muscle quality in the arm; $\mathrm{MQI}_{\mathrm{Leg}^{\prime}}$ muscle quality in the leg; $\mathrm{MQI}_{\text {Total' }}$ total muscle quality; $\mathrm{MQI}_{\mathrm{Std}}$, standardized muscle quality. 
Table 3

Sex- and race-adjusted Pearson partial correlations among muscle mass, muscle strength, and muscle quality in young and elderly adults

\begin{tabular}{|c|c|c|c|c|c|c|}
\hline & \multicolumn{3}{|c|}{ Young Adults } & \multicolumn{3}{|c|}{ Elderly Adults } \\
\hline & Muscle Mass & Muscle Strength & Muscle Quality & Muscle Mass & Muscle Strength & Muscle Quality \\
\hline Muscle Mass & 1.00 & 0.19 & $-0.48^{*}$ & 1.00 & 0.05 & $-0.73^{*}$ \\
\hline Muscle Strength & . & 1.00 & $0.62^{*}$ & . & 1.00 & $0.46^{*}$ \\
\hline Muscle Quality & . & . & 1.00 & . & . & 1.00 \\
\hline
\end{tabular}

\section{Discussion}

Although the rising trend in the prevalence of sarcopenia and disabilities is a major public health concern in the US (5-6), the accurate definition of sarcopenia still remains in controversial. To our knowledge, we first define sarcopenia using MQI cutpoints $\left(\mathrm{MQI}_{\mathrm{Arm}^{\prime}} \mathrm{MQI}_{\mathrm{Leg}^{\prime}} \mathrm{MQI}_{\mathrm{Total}^{\prime}}\right.$ and $\mathrm{MQI}_{\mathrm{Std}}$ ) based on young reference group. Several investigators have proposed muscle quality index or muscle power index using the ratio of muscle strength to muscle mass or the ratio of muscle power to muscle mass (24-25). BarbatArtigas et al. (24) proposed MQI cut-points using the ratio of grip strength to total skeletal muscle mass $(\mathrm{kg} /$ SMkg) based on young reference population. Using this ratio, they classified "poor" MQI cut-points in men and women as $\leq 1.36$ and $\leq 1.35 \mathrm{~kg} / \mathrm{SMkg}$. The Concord Health and Ageing in Men Project (CHAMP) proposed lower and upper extremity muscle quality scores based on the lowest $20 \%$ of the distribution in men aged 70 to 90 years (25). Other investigators have also proposed muscle power index using the ratio of muscle power (W) to total skeletal muscle mass $(\mathrm{SMkg})(24)$ or the ratio of muscle power to time (26). However, the feasibility of these indices in clinical settings as a screening tool to detect sarcopenia has not been well documented.

Muscle mass, muscle strength, and muscle quality are associated with physical function and disability, all of these are important factors to define sarcopenia (12, 14, 23, 24-25). The muscle quality represents muscle's ability to function, which is the best marker of functional capacity when compared with muscle mass or muscle strength (23). The CHAMP study also showed that muscle quality or muscle strength, not muscle mass, was a strong predictor of physical function and disability (25). Several International Working Groups have defined sarcopenia using the combination of low muscle mass and weakness or slowness (16-20). Defining sarcopenia using muscle mass and muscle weakness may have some limitations without considering myosteatosis (intermuscular and intramuscular adipose tissue). For instance, muscle mass or muscle strength with myosteatosis is not a good indicator of functional capacity because aging is positively associated with an increase in fatty infiltration of muscle tissue (27-28). Some investigators have also shown that elderly men had about $59-127 \%$ more fat in quadriceps and hamstrings than did young men (27), with an annual increase of intramuscular fat by $18 \%$ (28). Interestingly, an increase in fat mass is positively associated with muscle mass and muscle strength but is negatively associated with muscle quality (22). In fact, a greater muscle mass or muscle strength with excess fat may lack muscle quality, which may misclassify sarcopenic patients to nonsarcopenic patients. Increasing muscle strength per unit of muscle mass, not by accumulating fat mass, is associated with muscle quality. Our findings also show that muscle mass is inversely associated with muscle quality in young and elderly persons, which is consistent with the findings from the US general population and the French women study (29-30). The muscle quality is a strong surrogate marker for sarcopenia because it quantifies the function of muscle mass and muscle strength as a single unit. Further studies are needed to determine whether muscle quality is a better marker for physical disability and mortality as compared with the combination of low muscle mass and low muscle function, which defined sarcopenia by International Working Groups (16-20). Also, more studies are needed to justify the optimal cutpoints for MQIs, muscle strength, and muscle mass in relation to disability and mortality.

A strength of this study is that the MQIs are based on young healthy reference group. Our cut-points to define sarcopenia using ASM in men were slightly higher $\left(0.5 \mathrm{~kg} / \mathrm{m}^{2}\right)$ than those cut-points by Baumgartner et al. (12), but our women's cut-points were similar with the EWGSOP (16). In the $\mathrm{ASM}_{\mathrm{BMI}}$ cut-points, we observed that our ASMBMI cut-points were greater than the FNIH cut-points in both men and women, which may be due to methodological differences defining sarcopenia. For instance, the FNIH cut-points to define sarcopenia for $\mathrm{ASM}_{\mathrm{BMI}}$ were based on the elderly people (aged $70-90$ years) using the mean values of the lowest $20 \%$ distribution, whereas our cut-points to define sarcopenia 
were based on young reference group (aged 20-29 years) using $<2 \mathrm{SD}$ from the mean values. Another strength of our study is that we used DXA, a criterion method, to estimate lean mass in both arms and legs. A limitation of our study is that our findings may limit generalizability due to small sample size. Further studies are needed to define MQI cut-points with a large sample size across different race and gender groups.

In summary, muscle quality is a significant risk factor for disability and mortality. Based on healthy young reference men and women, we establish a new definition of sarcopenia using muscle quality indexes. At the very least, our muscle quality indices may still be a role as a screening tool in detecting sarcopenia across individual and population level.

Acknowledgements: This study was supported by Mayo-Arizona State University Obesity Solution Grant. The authors thank the graduate students from the ASU and study participants for their important contributions.

Conflict of Interest: None

Ethical standard: This study was performed in accordance with the ethical standards by the Arizona State University review board and with the 1964 Helsinki declaration and its later amendments or comparable ethical standards.

\section{References}

1. Landi F, Liperoti R, Russo A, et al. Sarcopenia as a risk factor for falls in elderly individuals: results from the ilSIRENTE study. Clin Nutr 2012;5:6528 .

2. Visser M, Goodpaster BH, Kritchevsky SB, et al. Muscle mass, muscle strength, and muscle fat infiltration as predictors of incident mobility limitations in well-functioning older persons. J Gerontol A Biol Sci Med Sci 2005;60:324-33.

3. Hirani V, Blyth F, Naganathan V, et al. Sarcopenia is associated with incident disability, institutionalization, and mortality in community-dwelling olde men: The Concord Health and Ageing in Men Project. J Am Med Dir Assoc 2015;16:607-613.

4. Shafiee G, Keshtkar A, Soltani A, Ahadi Z, Larijani B, Heshmat R. Prevalence of sarcopenia in the world: a systematic review and meta-analysis of general population studies. J Diabetes Metab Disord 2017;16:21

5. Janssen I, Heymsfield SB, Ross R. Low relative skeletal muscle mas (sarcopenia) in older persons is associated with functional impairment and physical disability. J Am Geriatr Soc 2002;50:889-96.

6. Batsis JA, Mackenzie TA, Lopez-Jimenez F, Bartels SJ. Sarcopenia, sarcopenic obesity, and functional impairments in older adults: National Health and Nutrition Examination Surveys 1999-2004. Nutr Res 2015;35:1031-9.

7. Morley JE, Anker SD, von Haehling S. Prevalence, incidence, and clinical impact of sarcopenia: facts, numbers, and epidemiology-update 2014. J Cachexia Sarcopenia Muscle 2014;5:253-9.

8. Psutka SP, Carrasco A, Schmit GD, et al. Sarcopenia in patients with bladder cancer undergoing radical cystectomy: impact on cancer-specific and allcause mortality. Cancer 2014;120:2910-8.

9. Lexell J, Taylor CC, Sjöström M. What is the cause of the ageing atrophy? Total number, size and proportion of different fiber types studied in whole vastus lateralis muscle from 15- to 83-year-old men. J Neurol Sci 1988;84:275-
94.

10. Doherty TJ. The influence of aging and sex on skeletal muscle mass and strength. Curr Opin Clin Nutr Metab Care 2001;4:503-8.

11. Batsis JA, Mackenzie TA, Barre LK, Lopez-Jimenez F, Bartels SJ. Sarcopenia sarcopenic obesity and mortality in older adults: results from the Nationa Health and Nutrition Examination Survey III. Eur J Clin Nutr 2014;68:1001-7.

12. Baumgartner RN, Koehler KM, Gallagher D, et al: Epidemiology of sarcopenia among the elderly in New Mexico. Am J Epidemiol 1998;147:75563

13. Srikanthan P, Karlamangla AS. Muscle mass index as a predictor of longevity in older adults. Am J Med 2014;127:547-53.

14. Visser M, Newman AB, Nevitt MC, Kritchevsky SB, Stamm EB, Goodpaster $\mathrm{BH}$, Harris TB. Reexamining the sarcopenia hypothesis. Muscle mass versus muscle strength. Health, Aging, and Body Composition Study Research Group. Ann N Y Acad Sci 2000;904:456-61.

15. Newman AB, Kupelian V, Visser M, et al. Strength, but not muscle mass, is associated with mortality in the health, aging and body composition study cohort. J Gerontol A Biol Sci Med Sci 2006;61:72-7.

16. Cruz-Jentoft AJ, Baeyens JP, Bauer JM, et al. Sarcopenia: European consensus on definition and diagnosis: Report of the European Working Group on Sarcopenia in Older People. Age Ageing. 2010 Jul;39(4):412-23.

17. Chen LK, Liu LK, Woo J, et al. Sarcopenia in Asia: consensus report of the Asian Working Group for Sarcopenia. J Am Med Dir Assoc 2014;15:95-101.

18. Fielding RA, Vellas B, Evans WJ, et al. Sarcopenia: an undiagnosed condition in older adults. Current consensus definition: prevalence, etiology, and consequences. International working group on sarcopenia. J Am Med Dir Assoc 2011;12:249-56.

19. Morley JE, Abbatecola AM, Argiles JM, et al. Sarcopenia with limited mobility: an international consensus. J Am Med Dir Assoc 2011;12:403-9.

20. Studenski SA, Peters KW, Alley DE, et al. The FNIH sarcopenia project: rationale, study description, conference recommendations, and final estimates. J Gerontol A Biol Sci Med Sci 2014;69:547-58.

21. Miljkovic I, Zmuda JM. Epidemiology of myosteatosis. Curr Opin Clin Nutr Metab Care 2010;13:260-4.

22. Koster A, Ding J, Stenholm S, et al. Does the amount of fat mass predict agerelated loss of lean mass, muscle strength, and muscle quality in older adults? J Gerontol A Biol Sci Med Sci 2011;66:888-95.

23. Misic MM, Rosengren KS, Woods JA, Evans EM. Muscle quality, aerobic fitness and fat mass predict lower-extremity physical function in communitydwelling older adults. Gerontology 2007;53:260-6.

24. Barbat-Artigas S, Rolland Y, Zamboni M, Aubertin-Leheudre M. How to assess functional status: a new muscle quality index. J Nutr Health Aging 2012;16:67-77.

25. Hairi NN, Cumming RG, Naganathan V, et al. Loss of muscle strength, mass (sarcopenia), and quality (specific force) and its relationship with functional limitation and physical disability: the Concord Health and Ageing in Men Project. J Am Geriatr Soc 2010;58:2055-62.

26. Takai Y, Ohta M, Akagi R, Kanehisa H, Kawakami Y, Fukunaga T. Sit-tostand test to evaluate knee extensor muscle size and strength in the elderly: a novel approach. J Physiol Anthropol 2009;28:123-8.

27. Overend TJ, Cunningham DA, Paterson DH, Lefcoe MS. Thigh composition in young and elderly men determined by computed tomography. Clin Physiol 1992;12:629-40.

28. Goodpaster BH, Chomentowski P, Ward BK, et al. Effects of physical activity on strength and skeletal muscle fat infiltration in older adults: a randomized controlled trial. J Appl Physiol 2008;105:1498-503.

29. Bouchard DR, Héroux M, Janssen I. Association between muscle mass, leg strength, and fat mass with physical function in older adults: influence of age and sex. J Aging Health 2011;23:313-28.

30. Barbat-Artigas S, Rolland Y, Vellas B, Aubertin-Leheudre M. Muscle quantity is not synonymous with muscle quality. J Am Med Dir Assoc 2013;14:852 e1-7. 